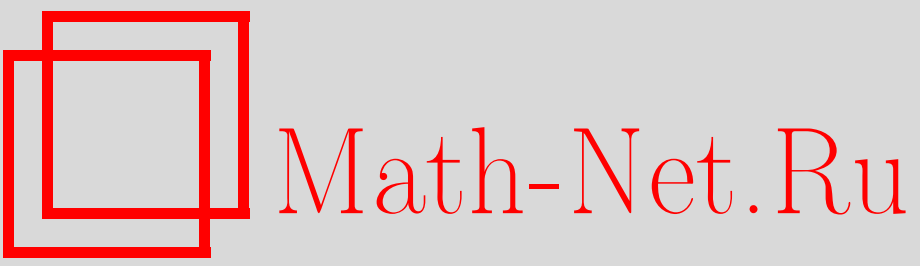

В. Н. Анисимов, В. Л. Литвинов, Исследование резонансных свойств механических объектов с движущимися границами при помощи метода КанторовичаГалёркина, Вестн. Сам. гос. техн. ун-та. Сер. Физ.-мат. науки, 2009, выпуск 1(), 149-158

DOI: https://doi.org/10.14498/vsgtu658

Использование Общероссийского математического портала Math-Net.Ru подразумевает, что вы прочитали и согласны с пользовательским соглашением http: //www. mathnet.ru/rus/agreement

Параметры загрузки:

IP: 54.81 .137 .203

26 апреля 2023 г., 08:17:37 
УДК 534.11

\section{ИССЛЕДОВАНИЕ РЕЗОНАНСНЫХ СВОЙСТВ МЕХАНИЧЕСКИХ ОБЪЕКТОВ С ДВИЖУЩИМИСЯ ГРАНИЦАМИ ПРИ ПОМОЩИ МЕТОДА КАНТОРОВИЧА-ГАЛЁРКИНА}

\section{В.Н. Анисимов, В.Л. Литвинов}

Сызранский филиал Самарского государственного технического университета, 446001, Самарская обл., Сызрань, ул. Советская, 45.

E-mails: anisimov170159@mail.ru, vladlitvinov@rambler.ru

Разработана обобщенная методика использования метода Канторовича в совокупности с методом Галёркина для исследования резонансных свойств механических систем с движущимися границами.

Ключевые слова: резонансные свойства, механические системы с движущимися границами, амплитуда колебаний.

В настоящее время вопросы надежности при проектировании машин и механизмов требуют все более полного учета динамических явлений, имеющих место в проектируемых объектах. Широкое распространение в технике имеют механические объекты с движущимися границами. Это канаты в грузоподъемных установках, ленты в лентопротяжных механизмах, звенья передач с гибкой связью, стержни твердого топлива при сгорании и т. д.

Точные методы решения таких задач ограничены волновым уравнением и сравнительно простыми граничными условиями [1]. Из приближенных методов наиболее эффективен метод Канторовича-Галёркина. Он использовался ранее в работе [2] для решения волнового уравнения и уравнения изгибных колебаний балки при несложных однородных граничных условиях, заданных на одной движущейся и одной неподвижной границах.

В данной работе метод распространен на более широкий класс задач, которые в случае неподвижных границ могут быть решены методом разделения переменных. Особое внимание уделено анализу получаемых решений на резонансные свойства. Произведена оценка точности метода в зависимости от скорости движения границ.

Пусть требуется получить решение дифференциального уравнения в частных производных

$$
U_{\tau \tau}(\xi, \tau)+L[U(\xi, \tau)]=\phi(\xi, \tau)
$$

при граничных условиях

$$
Y_{j i}\left[U\left(l_{j}(\varepsilon \tau), \tau\right)\right]=F_{j i}(\tau) ; \quad i=1,2, \ldots, m ; \quad j=1,2,
$$

где $L$ - линейный однородный дифференциальный оператор по переменной $\xi$ порядка $2 m ; Y_{j i}$ - линейные однородные дифференциальные операторы по $\xi$; $\phi(\xi, \tau), F_{j i}(\tau)$ - заданные функции; $\varepsilon-$ малый параметр (обычно величина $\varepsilon$ соизмерима с $v / a, v$-скорость границы, $a$-скорость распространения колебаний).

Анисимов Валерий Николаевич-доцент кафедры общетеоретических дисииплин; к.ф.-м.н., доиент.

Литвинов Владислав Лъвович - преподаватель кафедры общетеоретических дисциплин. 
Запись законов движения границ в виде $l_{j}(\varepsilon \tau)$ соответствует режиму медленного движения. Уравнение (1) и граничные условия (2)-самосопряжённые, и в случае неподвижности границ $\left(l_{j}(\varepsilon \tau)=\mathrm{const}\right)$ может быть получено точное решение методом разделения переменных.

Заметим, что метод Канторовича-Галёркина позволяет учесть и начальные условия. Однако цель статьи - анализ резонансных свойств, а начальные условия не влияют на резонансные свойства линейных систем, поэтому в задаче (1), (2) начальные условия опущены.

Для того чтобы избавиться от неоднородностей в граничных условиях, вводится новая функция

$$
U(\xi, \tau)=V(\xi, \tau)+H(\xi, \tau),
$$

где

$$
H(\xi, \tau)=\sum_{k=1}^{2} \sum_{r=1}^{m} D_{k r}(\xi, \varepsilon \tau) F_{k r}(\tau),
$$

а функция $D_{k r}(\xi, \varepsilon \tau)$ удовлетворяют уравнению

$$
L\left[D_{k r}(\xi, \varepsilon \tau)\right]=0
$$

и условиям

$$
Y_{j i}\left[D_{k r}\left(l_{j}(\varepsilon \tau), \tau\right)\right]= \begin{cases}1, & k=j \wedge r=i, \\ 0, & k \neq j \vee r \neq i .\end{cases}
$$

Решение задачи (4), (5) затруднений, как правило, не вызывает, и поэтому здесь она подробно не рассматривается.

Функция $V(\xi, \tau)$ находится как решение следующей задачи:

$$
\begin{gathered}
V_{\tau \tau}(\xi, \tau)+L[V(\xi, \tau)]=\phi(\xi, \tau)-H_{\tau \tau}(\xi, \tau), \\
Y_{j i}\left[V\left(l_{j}(\varepsilon \tau), \tau\right)\right]=0 .
\end{gathered}
$$

Для решения задачи используем метод Канторовича в совокупности с методом Галёркина. Решение задачи будем искать в виде

$$
V(\xi, \tau)=\sum_{n=1}^{\infty} f_{n}(\tau) X_{n}(\xi, \varepsilon \tau)
$$

где $X_{n}(\xi, \varepsilon \tau)$ - собственные функции следующей краевой задачи:

$$
\begin{gathered}
L\left[X_{n}(\xi, \varepsilon \tau)\right]=\omega_{0 n}^{2}(\varepsilon \tau) X_{n}(\xi, \varepsilon \tau) \\
Y_{j i}\left[X_{n}\left(l_{j}(\varepsilon \tau), \varepsilon \tau\right)\right]=0 .
\end{gathered}
$$

Здесь $\omega_{0 n}(\varepsilon \tau)$ - собственные частоты задачи. Оператор $L$ не содержит производной по $\tau$, поэтому величина $\varepsilon \tau$ рассматривается как параметр.

Такой выбор координатных функций $X_{n}$ обусловливает тот факт, что решение (7) является точным в случае, если границы неподвижны. При увеличении скорости движения границ точность метода будет уменьшаться. 
Заметим, что функции $X_{n}(\xi, \varepsilon \tau)$ удовлетворяют граничным условиям (6) и играют в данном случае роль динамических, т. е. изменяющихся со временем, мод.

Разложим функции $H(\xi, \tau)$ в ряд Фурье:

$$
H(\xi, \tau)=\sum_{n=1}^{\infty} \phi_{n}(\tau) X_{n}(\xi, \varepsilon \tau),
$$

где

$$
\phi_{n}(\tau)=\frac{\int_{l_{1}(\varepsilon \tau)}^{l_{2}(\varepsilon \tau)} H(\xi, \tau) X_{n}(\xi, \varepsilon \tau) q(\xi) d \xi}{\int_{l_{1}(\varepsilon \tau)}^{l_{2}(\varepsilon \tau)} X_{n}^{2}(\xi, \varepsilon \tau) q(\xi) d \xi} .
$$

Здесь $q(\xi)$ - весовая функция.

Тогда, согласно методу Галёркина, с учётом (8), (9), функции $f_{n}(\tau)$ будут удовлетворять следующей системе:

$$
\begin{array}{r}
\int_{l_{1}(\varepsilon \tau)}^{l_{2}(\varepsilon \tau)} \sum_{n=1}^{\infty}\left\{\left[f_{n}(\tau) X_{n}(\xi, \tau)\right]_{\tau \tau}+\omega_{n}^{2}(\varepsilon \tau) X_{n}(\xi, \varepsilon \tau) f_{n}(\tau)+\left[\phi_{n}(\tau) X_{n}(\xi, \varepsilon \tau)\right]_{\tau \tau}\right\} \times \\
\times X_{m}(\xi, \varepsilon \tau) q(\xi) d(\xi)=\int_{l_{1}(\varepsilon \tau)}^{l_{2}(\varepsilon \tau)} \phi(\xi, \tau) X_{m}(\xi, \varepsilon \tau) q(\xi) d(\xi), \quad(10)
\end{array}
$$

где $m \in \mathbb{N}$.

Решение системы (10) затруднительно. При резонансных явлениях амплитуды всех динамических мод, за исключением резонансной, малы. Поэтому в каждом уравнении системы членами, не содержащими $X_{m}(\xi, \varepsilon \tau)$, в связи с их малостью пренебрегают. В этом случае система (10) становится расщепленной и уравнение для нахождения $f_{n}(\tau)$ принимает вид

$$
\begin{aligned}
\int_{l_{1}(\varepsilon \tau)}^{l_{2}(\varepsilon \tau)}\left\{\left[\left[f_{n}(\tau)+\phi_{n}(\tau)\right]\right.\right. & \left.\left.X_{n}(\xi, \varepsilon \tau)\right]_{\tau \tau}+\omega_{n}^{2}(\varepsilon \tau) X_{n}(\xi, \varepsilon \tau) f_{n}(\tau)\right\} \times \\
\times X_{n}(\xi, \varepsilon \tau) q(\xi) d \xi & =\int_{l_{1}(\varepsilon \tau)}^{l_{2}(\varepsilon \tau)} \phi(\xi, \tau) X_{n}(\xi, \varepsilon \tau) q(\xi) d \xi .
\end{aligned}
$$

Введём новую функцию: $\mu_{n}(\tau)=f_{n}(\tau)+\phi_{n}(\tau)$, тогда уравнение (11) примет вид

$$
\begin{aligned}
A_{1 n}(\varepsilon \tau) \mu_{n}^{\prime \prime}(\tau)+2 \varepsilon A_{2 n}(\varepsilon \tau) \mu_{n}^{\prime}(\tau)+\varepsilon^{2} A_{3 n} & (\varepsilon \tau) \mu_{n}(\tau)+ \\
& +A_{1 n}(\varepsilon \tau) \omega_{n}^{2}(\varepsilon \tau) \mu_{n}(\tau)=\theta_{n}(\tau),
\end{aligned}
$$




$$
\begin{gathered}
\text { где } A_{1 n}(\varepsilon \tau)=\int_{l_{1}(\varepsilon \tau)}^{l_{2}(\varepsilon \tau)} X_{n}^{2}(\xi, \varepsilon \tau) q(\xi) d \xi ; \varepsilon A_{2 n}(\varepsilon \tau)=\int_{l_{1}(\varepsilon \tau)}^{l_{2}(\varepsilon \tau)} X_{n, \tau}(\xi, \varepsilon \tau) X_{n}(\xi, \varepsilon \tau) q(\xi) d \xi ; \\
\varepsilon^{2} A_{3 n}(\varepsilon \tau)=\int_{l_{1}(\varepsilon \tau)}^{l_{2}(\varepsilon \tau)} X_{n, \tau \tau}(\xi, \varepsilon \tau) X_{n}(\xi, \varepsilon \tau) q(\xi) d \xi ; \\
\theta_{n}(\tau)=\omega_{n}^{2}(\varepsilon \tau) A_{1 n}(\varepsilon \tau) \phi_{n}(\tau)+\int_{l_{1}(\varepsilon \tau)}^{l_{2}(\varepsilon \tau)} \phi(\xi, \tau) X_{n}(\xi, \varepsilon \tau) q(\xi) d \xi .
\end{gathered}
$$

С учётом (7), (9) решение (3) будет иметь вид

$$
U(\xi, \tau)=\sum_{n=1}^{\infty} \mu_{n}(\tau) X_{n}(\xi, \varepsilon \tau) .
$$

Данное решение показывает, что аналогично тому, как колебательные процессы для объектов с неподвижными границами выражаются суммой колебаний, соответствующих собственным функциям, так в случае движения границ появляется суперпозиция колебаний, соответствующих динамическим модам $X_{n}(\xi, \varepsilon \tau)$.

В большинстве практических задач границы движутся в медленном режиме и параметр $\varepsilon$ мал, поэтому в дальнейшем величины порядка $\varepsilon^{2}$ учитываться не будут.

Для упрощения введем в уравнение (12) новую функцию

$$
\mu_{n}(\tau)=A_{0 n}(\varepsilon \tau) y_{n}(\tau),
$$

где

$$
A_{0 n}(\varepsilon \tau)=\exp \left[-\int_{0}^{\tau} \frac{\varepsilon A_{2 n}(\varepsilon \zeta)}{A_{1 n}(\varepsilon \zeta)} d \zeta\right] .
$$

В этом случае уравнение (12) с точностью до величин порядка $\varepsilon^{2}$ будет иметь вид

$$
y_{n}^{\prime \prime}(\tau)+\omega_{n}^{2}(\varepsilon \tau) y_{n}(\tau)=\frac{\theta_{n}(\tau)}{A_{0 n}(\varepsilon \tau) A_{1 n}(\varepsilon \tau)} .
$$

Пусть внешнее воздействие на систему носит гармонический характер, T. e.

$$
\begin{gathered}
\phi(\xi, \tau)=B_{0}(\xi) \cos W_{0}(\tau) \\
F_{j i}(\tau)=B_{j i} \cos W_{j i}(\tau) ; \quad j=1,2 ; \quad i=1,2, \ldots, m,
\end{gathered}
$$

где $B_{0}(\xi)$ - функция, характеризующая интенсивность распределённой нагрузки; $W_{0}(\tau), W_{j i}(\tau)$ - монотонно возрастающие функции; $B_{j i}$ - постоянные величины. 
Ограничимся рассмотрением случая, когда правую часть уравнения (14) можно представить в виде

$$
\frac{\theta_{n}(\tau)}{A_{0 n}(\varepsilon \tau) A_{1 n}(\varepsilon \tau)}=M_{n}(\varepsilon \tau) \cos W_{n}(\tau)
$$

где $W_{n}(\tau)$ - монотонно возрастающая функция.

Представление (15) возможно в следующих случаях: 1) все внешние возмущения равны нулю кроме какого-то одного; 2$)$ функции $W_{0}(\tau), W_{j i}(\tau)$ отличаются на постоянную величину; 3) резонансные области внешних нагрузок не пересекаются, тогда при рассмотрении резонанса от одной нагрузки действием других можно пренебречь.

С учётом изложенного уравнение (14) примет вид

$$
y_{n}^{\prime \prime}(\tau)+\omega_{n}^{2}(\varepsilon \tau) y_{n}(\tau)=M_{n}(\varepsilon \tau) \cos W_{n}(\tau) .
$$

Решение данного уравнения при начальных условиях $y(0)=0, y^{\prime}(0)=0$ записывается следующим образом [3]:

$$
y_{n}(\tau)=\int_{0}^{\tau} \gamma_{n}(\tau, \zeta) M_{n}(\varepsilon \zeta) \cos W_{n}(\zeta) d \zeta,
$$

где

$$
\gamma_{n}(\tau, \zeta)=\frac{y_{1 n}(\tau) y_{2 n}(\zeta)-y_{1 n}(\zeta) y_{2 n}(\tau)}{y_{1 n}(\zeta) y_{2 n}^{\prime}(\zeta)-y_{1 n}^{\prime}(\zeta) y_{2 n}(\zeta)}
$$

a $y_{1 n}, y_{2 n}$-линейно независимые решения однородного уравнения, соответствующего (16).

С помощью метода малого параметра [4] с точностью до величин порядка $\varepsilon^{2}$ найдём:

$$
y_{1 n}(\tau)=a_{n}(\varepsilon \tau) \sin w_{n}(\tau) ; \quad y_{2 n}(\tau)=a_{n}(\varepsilon \tau) \cos w_{n}(\tau),
$$

где функции $a_{n}(\varepsilon \tau)$ и $w_{n}(\tau)$ определяются из системы уравнений

$$
\left\{\begin{array}{l}
\frac{d w_{n}(\tau)}{d \tau}=\omega_{n}(\varepsilon \tau) \\
\frac{d a_{n}(\varepsilon \tau)}{d \tau}=-\frac{a_{n}(\varepsilon \tau)}{2 \omega_{n}(\varepsilon \tau)} \frac{d \omega_{n}(\varepsilon \tau)}{d \tau},
\end{array}\right.
$$

решение которой даёт

$$
w_{n}(\tau)=\int_{0}^{\tau} \omega_{n}(\varepsilon \tau) d \tau ; \quad a_{n}(\varepsilon \tau)=\frac{1}{\sqrt{\omega_{n}(\varepsilon \tau)}} .
$$

Возвращаясь к решению (17), с учётом (18) получим: 


$$
\begin{aligned}
y_{n}(\tau)=a_{n}(\varepsilon \tau) \sin w_{n}(\tau) & \int_{0}^{\tau} \frac{M_{n}(\varepsilon \zeta) \cos W_{n}(\zeta) \cos w_{n}(\zeta)}{a_{n}(\varepsilon \zeta) w_{n}^{\prime}(\zeta)} d \zeta- \\
& -a_{n}(\varepsilon \tau) \cos w_{n}(\tau) \int_{0}^{\tau} \frac{M_{n}(\varepsilon \zeta) \cos W_{n}(\zeta) \sin w_{n}(\zeta)}{a_{n}(\varepsilon \zeta) w_{n}^{\prime}(\zeta)} d \zeta .
\end{aligned}
$$

Разлагая произведение тригонометрических функций в сумму и учитывая замену (13), можно получить следующее выражение для полной амплитуды колебаний, соответствующих $n$-ной динамической моде:

$$
\begin{aligned}
A_{n}^{2}(\tau)=\frac{1}{4} A_{0 n}^{2}(\varepsilon \tau) a_{n}^{2}(\varepsilon \tau)\left\{\left[\int_{0}^{\tau} F_{n}(\varepsilon \zeta) \cos \Phi_{n 1}(\zeta) d \zeta+\int_{0}^{\tau} F_{n}(\varepsilon \zeta) \cos \Phi_{n 2}(\zeta) d \zeta\right]^{2}+\right. \\
\left.+\left[\int_{0}^{\tau} F_{n}(\varepsilon \zeta) \sin \Phi_{n 1}(\zeta) d \zeta+\int_{0}^{\tau} F_{n}(\varepsilon \zeta) \sin \Phi_{n 2}(\zeta) d \zeta\right]^{2}\right\}
\end{aligned}
$$

где $F_{n}(\varepsilon \zeta)=\frac{M_{n}(\varepsilon \zeta)}{a_{n}(\varepsilon \zeta) w_{n}(\zeta)} ; \Phi_{n 1}(\zeta)=w_{n}(\zeta)-W_{n}(\zeta) ; \Phi_{n 2}(\zeta)=w_{n}(\zeta)+W_{n}(\zeta)$. Здесь функция $F_{n}(\varepsilon \zeta)$ знакопостоянна, так как функции $M_{n}(\varepsilon \zeta)$ положительны, а произведение $a_{n}(\varepsilon \zeta) w_{n}^{\prime}(\zeta)$ знакопостоянно (оно равно якобиану двух линейно независимых функций $y_{1 n}$ и $\left.y_{2 n}\right)$. Функции $w_{n}(\zeta)$ и $W_{n}(\zeta)$ монотонно возрастают, поэтому фаза $\Phi_{n 2}(\zeta)$ изменяется быстрее фазы свободных колебаний, которая определяется функцией $w_{n}(\zeta)$. Следовательно, участок знакопостоянства функций $\sin \Phi_{n 2}(\zeta), \cos \Phi_{n 2}(\zeta)$ меньше половины периода свободных колебаний, т. е. период возрастания соответствующих интегралов невелик. Интегралы же, содержащие $\sin \Phi_{n 1}(\zeta), \cos \Phi_{n 1}(\zeta)$, возрастают в течение всего периода, пока наблюдается резонансное явление, и вносят основной вклад в амплитуду. Пренебрегая членами, содержащими $\Phi_{n 2}(\zeta)$, получим следующее выражение для амплитуды колебаний:

$$
\begin{aligned}
A_{n}^{2}(\tau)=\frac{1}{4} A_{0 n}^{2}(\varepsilon \tau) a_{n}^{2}(\varepsilon \tau)\left\{\left[\int_{0}^{\tau} F_{n}(\varepsilon \zeta) \cos \Phi_{n 1}(\zeta) d \zeta\right]^{2}+\right. \\
\left.+\left[\int_{0}^{\tau} F_{n}(\varepsilon \zeta) \sin \Phi_{n 1}(\zeta) d \zeta\right]^{2}\right\} .
\end{aligned}
$$

Полученное выражение удобно для анализа резонансных свойств систем с движущимися границами. В таких системах различают два вида резонансных явлений: установившийся резонанс и прохождение через резонанс.

Установившийся резонанс - это явление резкого увеличения амплитуды колебаний в случае, когда изменение частоты внешней силы и одной из собственных частот согласованы таким образом, что создаются наилучшие условия для возрастания амплитуды. 
Прохождение через резонанс - это явление резкого увеличения амплитуды в течение конечного промежутка времени, когда мгновенная частота одного из собственных колебаний проходит через значение возмущающей частоты.

Заметим, что при стремлении скорости движения границ к нулю явление установившегося резонанса и явление прохождения через резонанс вырождаются в явления обычного резонанса для системы с неподвижными границами.

Из выражения (19) следует, что установившийся резонанс будет наблюдаться, если $\Phi_{n 1}(\zeta)=\gamma=$ const.

Явление прохождения через резонанс наблюдается во временной области, содержащей точку $\tau_{0}$, где $\Phi_{n 1}^{\prime}\left(\tau_{0}\right)=0$. В этой точке мгновенная частота $n$ ного собственного колебания проходит через значение возмущающей частоты. Прохождение через резонанс начинается не доходя до точки $\tau_{0}$ и заканчивается за этой точкой. Если принять амплитуду в начале резонансной области (точка $\tau_{1}$ ) равной нулю, то амплитуда в конце резонансной области (точка $\left.\tau_{2}\right)$ будет определяться следующим выражением:

$$
\begin{aligned}
A_{n}^{2}\left(\tau_{1} ; \tau_{2}\right)=E_{n}^{2}\left(\tau_{2}\right)\left\{\left[\int_{\tau_{1}}^{\tau_{2}} F_{n}(\varepsilon \zeta) \cos \Phi_{n 1}(\zeta) d \zeta\right]^{2}+\right. & \\
& \left.+\left[\int_{\tau_{1}}^{\tau_{2}} F_{n}(\varepsilon \zeta) \sin \Phi_{n 1}(\zeta) d \zeta\right]^{2}\right\}
\end{aligned}
$$

Исследование прохождения через резонанс заключается в определении границ резонансной области $\tau_{1}$ и $\tau_{2}$, соответствующих максимуму выражения (20), причём $\tau_{1}<\tau_{0}$, а $\tau_{2}>\tau_{0}$.

В качестве примера рассмотрим вынужденные колебания струны с равномерно движущейся границей. Зависимость силы сопротивления движению струны примем пропорциональной её скорости. Тогда дифференциальное уравнение, описывающее колебания струны, будет иметь вид

$$
\rho Z_{t t}(x, t)-T Z_{x x}(x, t)+\lambda Z_{t}(x, t)=0,
$$

где $\rho$ - линейная плотность массы струны; $T$ - сила натяжения струны; $\lambda-$ сила сопротивления среды, действующая на единицу длины струны при единичной скорости поперечного движения; $Z(x, t)$ - поперечное смещение точки струны с координатой $x$ в момент времени $t$.

Рассмотрим граничное условие следующего вида:

$$
Z(0, t)=0 ; \quad Z\left(l_{0}(t), t\right)=B \cos W_{0}\left(\omega_{0} t\right),
$$

где $l_{0}(t)=L_{0}+v_{0} t-$ закон движения границы; $W_{0}\left(\omega_{0} t\right)-$ монотонно возрастающая функция; $B, \omega_{0}$ - постоянные величины (в случае действия гармонического возмущения $\omega_{0}$ является частотой возмущения).

Введём в задачу (21), (22) безразмерные переменные:

$$
\xi=\omega_{0} \frac{x}{a}, \quad \tau=\omega_{0} t+\frac{\omega_{0} L_{0}-a}{v_{0}}, \quad Z(x, t)=B z(\xi, \tau)
$$


и новую функцию $z(\xi, \tau)=\exp (-\alpha \tau) U(\xi, \tau)$, где $a=\sqrt{\frac{T}{\rho}} ; \alpha=\frac{\lambda}{2 \omega_{0} \rho}$.

Тогда после преобразований получим:

$$
\begin{gathered}
U_{\tau \tau}(\xi, \tau)-U_{\xi \xi}(\xi, \tau)-\alpha^{2} U(\xi, \tau)=0 \\
U(0, \tau)=0 ; \quad U(l(\varepsilon, \tau), \tau)=\exp (\alpha \tau) \cos W(\tau) .
\end{gathered}
$$

Здесь использованы следующие обозначения:

$$
l(\varepsilon, \tau)=1+\varepsilon \tau, \quad \varepsilon=\frac{V_{0}}{a}, \quad W(\tau)=W_{0}\left(\tau+\gamma_{0}\right), \quad \gamma_{0}=\frac{a-\omega_{0} L_{0}}{v_{0}} .
$$

Заметим, что величина $\exp (-2 \pi \alpha)$ характеризует относительное изменение амплитуды свободных колебаний за одно собственное колебание, и $\alpha$ в большинстве случаев является величиной того же порядка малости, что и $\varepsilon$.

Для решения задачи используем описанную выше методику.

В результате получим следующее выражение для амплитуды колебаний, соответствующих $n$-ной динамической моде:

$$
A_{n}^{2}(\tau)=E_{n}^{2}(\tau)\left\{\left[\int_{0}^{\tau} F_{n}(\zeta) \cos \Phi_{n}(\zeta) d \zeta\right]^{2}+\left[\int_{0}^{\tau} F_{n}(\zeta) \sin \Phi_{n}(\zeta) d \zeta\right]^{2}\right\}
$$

где

$$
\begin{gathered}
\omega_{n}(\varepsilon \tau)=\frac{\pi^{2} n^{2}}{l(\varepsilon \tau)}-\alpha^{2} ; \quad w_{n}(\tau)=\frac{\pi n}{\varepsilon}\left[S(\varepsilon \tau)-\frac{1}{2} \ln \frac{1+S(\varepsilon \tau)}{1-S(\varepsilon \tau)}\right] ; \\
S(\varepsilon \tau)=\sqrt{1-\left(\frac{\alpha(1+\varepsilon \tau)}{\pi n}\right)^{2}} ; \quad E_{n}^{2}(\tau)=\frac{\exp (-2 \alpha \tau)}{4 l(\varepsilon \tau) \omega_{n}(\varepsilon \tau)} \\
\Phi_{n}(\zeta)=w_{n}(\zeta)-W(\zeta) ; \quad F_{n}(\zeta)=\frac{(-1)^{n+1} 2 \pi n \exp (\alpha \zeta)}{\sqrt{\omega_{n}(\varepsilon \zeta) l^{3}(\varepsilon \zeta)}}
\end{gathered}
$$

Установившийся резонанс в рассматриваемой системе наблюдается, если

$$
W(\zeta)=w_{n}(\zeta)+\gamma,
$$

где $\gamma$ - постоянная величина. Амплитуда при этом имеет вид

$$
A_{n}(\tau)=\exp (-\alpha \tau) \int_{0}^{\tau} \frac{\exp (\alpha \zeta)}{1+\varepsilon \zeta} d \zeta
$$

Численное исследование этого выражения показывает, что при уменьшении длины струны $(\varepsilon<0)$ амплитуда колебаний непрерывно возрастает. При увеличении длины $(\varepsilon>0)$ амплитуда сначала возрастает, достигая некоторого максимального значения, а потом начинает убывать. Убывание амплитуды связано с тем, что демпфирующие силы, начиная с некоторого момента времени, начинают преобладать над возмущающими, так как длина колеблющейся части увеличивается.

Зависимость максимальной амплитуды от $\alpha$ и $\varepsilon$ (от номера $n$ она не зависит) приведена в таблице. 


\begin{tabular}{c|c|c|c|c|c}
\hline \multirow{2}{*}{$\alpha$} & \multicolumn{5}{|c}{$\varepsilon$} \\
\cline { 2 - 6 } & 0,001 & 0,005 & 0,010 & 0,100 & 0,200 \\
\hline 0,00 & 1000 & 201 & 101 & 10,1 & 5,1 \\
\hline 0,05 & 51,3 & 27,1 & 20,1 & 5,4 & 3,3 \\
\hline 0,10 & 31,5 & 17,3 & 13,4 & 4,2 & 2,7 \\
\hline
\end{tabular}

Исследуем явление прохождения через резонанс, возникающее в струне под действием силы постоянной частоты, т. е. $W(\tau)=\tau$, что в исходной системе соответствует действию силы с частотой $\omega_{0}$.

Максимально возможная амплитуда колебаний совпадает с максимумом выражения

$$
A_{n}^{2}\left(\tau_{1}, \tau_{2}\right)=E_{n}^{2}\left(\tau_{2}\right)\left\{\left[\int_{\tau_{1}}^{\tau_{2}} F_{n}(\zeta) \cos \Phi_{n}(\zeta) d \zeta\right]^{2}+\left[\int_{\tau_{1}}^{\tau_{2}} F_{n}(\zeta) \sin \Phi_{n}(\zeta) d \zeta\right]^{2}\right\} .
$$

Прохождение через резонанс начинается не доходя до точки $\tau_{0}\left(\tau_{1}<\tau_{0}\right)$ и заканчивается за этой точкой $\left(\tau_{2}>\tau_{0}\right)$. Сама точка $\tau_{0}$ определяется по следующей формуле:

$$
\tau_{0}=\frac{1}{\varepsilon}\left(\frac{\pi n}{\sqrt{1+\alpha^{2}}}-1\right)
$$

В результате исследования прохождения через резонанс получена зависимость максимальной амплитуды колебаний, возникающих при прохождении через резонанс на первой динамической моде, от $\alpha$ и $\varepsilon$. Данная зависимость приведена на рисунке.

Анализ графика показывает, что амплитуда при $\alpha=0$ является оценкой сверху для амплитуды колебаний, возникающих при прохождении через резонанс, когда $\alpha>0$. Когда действие демпфирующих сил не учитывается $(\alpha=0)$, задача (23), (24) может быть решена точным методом. Это сделано в работе [5]. Срав-

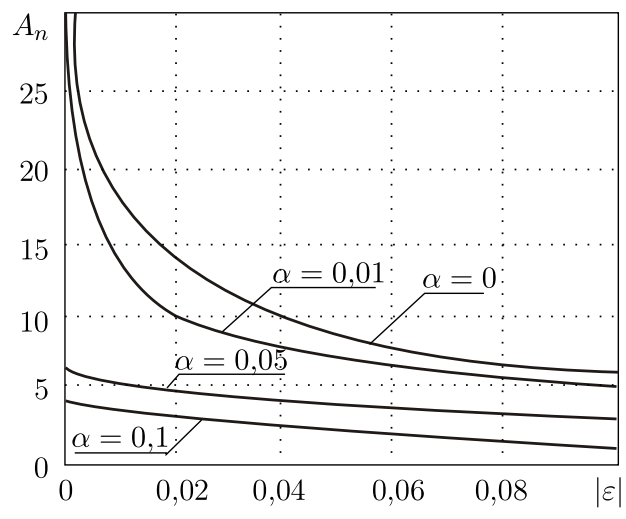

Зависимость амплитуды колебаний струны от $\alpha$ и $\varepsilon$ при прохождении через резонанс на первой динамической моде нение результатов показывает, что метод Канторовича-Галёркина даёт в рассматриваемом случае $(\varepsilon<0,1)$ удовлетворительные по точности результаты (погрешность лежит в пределах $5 \%)$.

\section{БИБЛИОГРАФИЧЕСКИЙ СПИСОК}

1. Весницкий А.И. Волны в системах с движущимися границами и нагрузками. - М.: Физматлит, 2001. - 320 с.

2. Лежнева А. А. Изгибные колебания балки переменной длины// Изв. АН CCCP. MTT, 1970. - C. $73-81$. 
3. Камке Э. Справочник по обыкновенным дифференциальным уравнениям. - М.: Наука, 1976. - $576 \mathrm{c}$.

4. Митропольский Ю. А. Проблемы асимптотической теории нестационарных колебаний. М.: Наука, 1964. - 432 с.

5. Анисимов В.Н. Исследование резонансных свойств одномерных механических систем с движущимися границами. - Куйбышев: КПтИ, 1985. - 18 с. - Деп. в ВИНИТИ 03.07.85, № $807-85$.

Поступила в редакцию 15/I/2009;

в окончательном варианте - 16/II/2009.

MSC: 74H45, 74K05

INVESTIGATION OF RESONANCE CHARACTERISTICS OF
MECHANICAL OBJECTS WITH MOVING BORDERS BY
APPLICATION OF THE KANTOROVICH-GALYORKIN METHOD

\section{V.N.Anisimov, V.L. Litvinov}

Syzran' Branch of Samara State Technical University 45, Sovetskaya st., Syzran', Samara region, 446001.

E-mails: anisimov170159@mail.ru, vladlitvinov@rambler.ru

Generalized procedure of the Kantorovich-Galyorkin method application for studying of resonance characteristics of mechanical systems with moving borders was developed.

Key words: resonance characteristics, mechanical systems with moving borders, amplitude vibrations.

Original article submitted 15/I/2009; revision submitted $16 / \mathrm{II} / 2009$.

Anisimov Valeriy Nikolayevich, Ph. D. (Phys. \& Math.), Ass. Prof., Dept. of generaltheoretical disciplines.

Litvinov Vladislav Lvovich, Teacher, Dept. of general-theoretical disciplines. 